Groups Geom. Dyn. 8 (2014), 441-466

DOI $10.4171 / \mathrm{GGD} / 233$
Groups, Geometry, and Dynamics

(C) European Mathematical Society

\title{
Optimal higher-dimensional Dehn functions for some CAT(0) lattices
}

\author{
Enrico Leuzinger
}

\begin{abstract}
Let $X=S \times E \times B$ be the metric product of a symmetric space $S$ of noncompact type, a Euclidean space $E$ and a product $B$ of Euclidean buildings. Let $\Gamma$ be a discrete group acting isometrically and cocompactly on $X$. We determine a family of quasi-isometry invariants for such $\Gamma$, namely the $k$-dimensional Dehn functions, which measure the difficulty to fill $k$ spheres by $(k+1)$-balls (for $1 \leq k \leq \operatorname{dim} X-1$ ). Since the group $\Gamma$ is quasi-isometric to the associated $\mathrm{CAT}(0)$ space $X$, assertions about Dehn functions for $\Gamma$ are equivalent to the corresponding results on filling functions for $X$. Basic examples of groups $\Gamma$ as above are uniform $S$-arithmetic subgroups of reductive groups defined over global fields. We also discuss a (mostly) conjectural picture for non-uniform $S$-arithmetic groups.
\end{abstract}

Mathematics Subject Classification (2010). 20F67, 20F69, 20E42, 53C35.

Keywords. Filling functions, higher-dimensional Dehn functions, isoperimetric inequalities, symmetric spaces, Euclidean buildings, lattices, $S$-arithmetic groups.

\section{Introduction and main results}

Since Gromov's seminal essay [26] the study of quasi-isometry invariants of (Cayley graphs of) finitely generated groups is of central interest in geometric group theory. Dehn functions are basic and well-studied examples of such quasi-isometry invariants. They measure the difficulty to fill circles by discs and are closely related to the complexity of the word problem (see e.g. [12]). For example, the Dehn function of a CAT(0) group is either linear or quadratic. A finitely generated group is Gromovhyperbolic if and only if its Dehn function is linear (see [12], 6.1.5).

More generally, higher-dimensional homotopical resp. homological Dehn (or filling) functions are quasi-isometry invariants which encode the difficulty to fill $k$ spheres by $(k+1)$-balls resp. $k$-cycles by $(k+1)$-chains. They were first considered in [20] and [26] $\$ 5$, and then more systematically in [3]. In contrast to the classical 1-dimensional Dehn functions much less is known about higher-dimensional Dehn functions. Recently, versions of such quasi-isometry invariants have been investigated by several authors for various classes of finitely generated groups (see e.g. [1], [11], [24], [41], [49], [51], [52]). 
The chief goal of the present paper is Theorem 2, in which we precisely determine the higher-dimensional Dehn functions for groups $\Gamma$ acting geometrically (i.e., isometrically, properly discontinuously and cocompactly) on a product $X=S \times E \times B$, where $S=G / K$ is a symmetric space of noncompact type (with $G$ a semisimple, noncompact real Lie group and $K \subset G$ a maximal compact subgroup), $E$ is a Euclidean space and $B$ is a product of Euclidean buildings. These higher-dimensional Dehn functions are (essentially) equivalent to the corresponding filling functions of the associated complete simply connected $\mathrm{CAT}(0)$ space $X$, which we compute in Theorem 1.

Examples of groups $\Gamma$ for which Theorem 2 holds are crystallographic groups, uniform lattices (arithmetic or not) in semisimple real Lie groups and (uniform) $S$ arithmetic subgroups of connected, reductive and $\mathbb{K}$-anisotropic $\mathbb{K}$-groups (where $\mathbb{K}$ is a global field). We briefly discuss this last family in Section 4. Our results are motivated by the corresponding problem to determine the higher-dimensional Dehn functions for $S$-arithmetic subgroups of connected, reductive and $\mathbb{K}$-isotropic $\mathbb{K}$-groups, which yield non-uniform lattices acting on $\operatorname{CAT}(0)$ spaces $X$ as above. Since such a non-uniform lattice is not quasi-isometric to $X$, this latter problem is much more difficult. In Section 5 we solve it for arbitrary lattices in $\operatorname{SO}(n, 1)$ and $\mathrm{SU}(n, 1)$ (see Theorems 4 and 5). We also provide a (mostly) conjectural picture for the general case (see Conjecture 1).

Beside hyperbolic groups studied in [37], [32] and Heisenberg groups treated in [51], the groups $\Gamma$ covered by Theorems 2, 4 and 5 seem currently to be the only examples of families of finitely generated discrete groups whose Dehn functions are known in all dimensions.

1.1. Higher-dimensional filling functions for some CAT(0) spaces. In order to state our results we first recall the concept of higher-dimensional filling functions for CAT(0) spaces $X=S \times E \times B$ as above. Consider an (integral) Lipschitz $k$-chain in $X$, i.e., a finite linear combination $\Sigma=\sum_{i} a_{i} \sigma_{i}$, with $a_{i} \in \mathbb{Z}$ and $\sigma_{i}: \Delta^{k} \rightarrow X$ a Lipschitz map from the $k$-dimensional Euclidean standard simplex to $X$. Note that $S$ and $E$ are smooth manifolds and $B$ is a piecewise Euclidean (poly)-simplicial complex with bounded geometry. Therefore, by Rademacher's theorem ([21], 3.1.6), such a $\sigma_{i}$ is differentiable almost everywhere and we thus can define $\operatorname{vol}_{k} \sigma_{i}$ as the integral over its Jacobian (for smooth maps this is the Riemannian volume of the image, compare [15], 5.5). We then define the $k$-volume or $k$-mass of $\Sigma$ as

$$
\operatorname{vol}_{k} \Sigma:=\sum_{i}\left|a_{i}\right| \operatorname{vol}_{k} \sigma_{i}
$$

We wish to measure the difficulty to fill Lipschitz $k$-cycles by Lipschitz $(k+1)$ chains. More precisely, for an integral Lipschitz $k$-cycle $\Sigma$ we define its filling volume

$$
\mathrm{FVol}_{k+1}(\Sigma):=\inf \left\{\operatorname{vol}_{k+1} \Omega \mid \Omega=\operatorname{Lipschitz}(k+1) \text {-chain with } \partial \Omega=\Sigma\right\} .
$$


Notice that the CAT( 0 ) space $X=S \times E \times B$ is contractible and thus $k$-connected for any $0 \leq k \leq n-1=\operatorname{dim} X-1$ (i.e., all homotopy groups $\pi_{k}(X)$ are trivial); hence the corresponding homology groups are also trivial (by the Hurewicz isomorphism theorem) and such fillings exist.

The $(k+1)$-dimensional filling function of $X$ is then given by

$$
\mathrm{FV}_{X}^{k+1}(l):=\sup \left\{\mathrm{FVol}_{k+1}(\Sigma) \mid \Sigma=\text { Lipschitz } k \text {-cycle in X with } \operatorname{vol}_{k} \Sigma \leq l\right\} .
$$

We emphasize that we are interested in the asymptotic geometry of $X$, i.e., the cycles we wish to fill are supposed to be "large". In fact, volumes of "small" domains have Euclidean behaviour and in particular satisfy superlinear isoperimetric inequalities (see [27], p. 322).

Quasi-isometry invariants are obtained from filling functions by considering equivalence classes for the following (standard) equivalence relation. Let $f, g: \mathbb{R} \rightarrow \mathbb{R}$ be two functions. We write $f \prec g$, if there is a constant $C>0$ such that $f(x) \leq C g(C x+C)+C x+C$ and $f \sim g$, if $f \prec g$ and $g \prec f$. Our key result is the following.

Theorem 1 (Filling functions). Let $X=S \times E \times B$ be the metric product of a symmetric space of noncompact type $S=G / K$, a Euclidean space $E$ and a product $B$ of Euclidean buildings (one or two factors of $X$ may be trivial). Let $\operatorname{rank} X=\operatorname{rank}_{\mathbb{R}} G+\operatorname{dim} E+\operatorname{dim} B$ be the Euclidean rank of $X$. For $k \geq 1$, let $\mathrm{FV}_{X}^{k+1}$ be the $(k+1)$-dimensional filling volume function of $X$. Then

(i) $X$ has Euclidean filling functions below the rank:

$$
\mathrm{FV}_{X}^{k+1}(l) \sim l^{\frac{k+1}{k}} \quad \text { if } k \leq \operatorname{rank} X-1 ;
$$

(ii) $X$ has linear filling functions above the rank:

$$
\mathrm{FV}_{X}^{k+1}(l) \sim l \quad \text { if } \operatorname{rank} X \leq k \leq \operatorname{dim} X-1 .
$$

Notice that case (ii) in Theorem 1 only occurs if the symmetric factor $S$ is nontrivial.

If $X=S$ is a symmetric space of noncompact type, Theorem 1 has been correctly asserted by Gromov in [26], 5.D (5) ( $\left.b^{\prime}\right)$. He also proposed a possible proof for an upper bound via isoperimetric inequalities: Pick some maximal flat $F$ in $X$ and project orthogonally to that flat. Then use the resulting cylinder to produce the desired filling. Gromov claims that this projection exponentially retracts the volume (as a function of the distance $d$ to $F$ ). In $6 . \mathrm{B}_{2}\left(\mathrm{~d}^{\prime \prime}\right)$ he attributes this to Mostow. But Mostow actually only proves (using quite delicate estimates) that the contraction is proportional to $d^{-1 / 2}$ when restricted to an $r$-dimensional submanifold (see [38], Lemma 6.4). The problem is that vectors tangent to singular geodesics can have projections with contraction factor almost equal to one, so that it is not clear whether 
this idea works. Nevertheless our approach is still quite similar: instead of projecting to a flat we project to a suitable horosphere.

A result of Wenger [47] asserts that a complete, simply connected CAT(0) space satisfies Euclidean isoperimetric inequalities in all dimensions (see Proposition 1 below). This yields Euclidean upper bounds for filling functions (see Section 2.1). Theorem 1 shows that this inequalities are sharp below the rank. Moreover it refines Wenger's general result in the present setting for dimensions above the rank (again showing that linear isoperimetric inequalities are sharp by providing lower bounds). In particular, Theorem 1 asserts that the filling functions of a symmetric space detect its rank.

Hyperbolic spaces satisfy linear isoperimetric inequalities in all dimensions. Theorem 1 thus also goes with Gromov's philosophy concerning the notion of "rank" in non-positive curvature as expressed in [26], 6. $\mathrm{B}_{2}$ :

One of the guiding principles in the asymptotic geometry of spaces $X$ with $K \leq 0$ can be expressed as follows. All flatness of $X$ where $K=0$ is confined to $k$-flats in $X$... One distinguishes among them maximal flats ... and then tries to show that $X$ is "hyperbolic transversally to maximal flats".

1.2. Higher-dimensional Dehn functions for some CAT(0) groups. We now pass from spaces to groups. Recall that a group $\Gamma$ is of type $F_{m}$, if there exists an EilenbergMac Lane complex $K(\Gamma, 1)$ with finite $m$-skeleton. A group is of type $F_{\infty}$, if it is of type $F_{m}$ for all $m$. The finiteness properties $F_{1}$ and $F_{2}$ are equivalent to $\Gamma$ being finitely generated and finitely presented, respectively. Alonso showed in [Al] that "being of type $F_{m}$ " is invariant under quasi-isometries.

Suppose that a given group $\Gamma$ is of type $F_{k+1}$. Then there exists a $k$-connected $(k+1)$-complex $K$ on which $\Gamma$ acts cocompactly (see [13] and [41]). A combinatorial map $\Sigma: S^{k} \rightarrow K^{(k)}$ of the standard $k$-sphere into the $k$-skeleton of $K$ can then be filled by a combinatorial $(k+1)$-ball $\Omega$. We define the combinatorial $k$-volume of $\Sigma$ as the number of non-degenerate $k$-cells in the image

$$
\operatorname{vol}_{k}^{c}(\Sigma):=\sharp\left\{\sigma \subset \Sigma\left(S^{k}\right) \mid \sigma \text { is a non-degenerate } k \text {-cell of } K\right\} .
$$

Analogously we define the combinatorial $(k+1)$-volume of $\Omega$ as the number of non-degenerate $(k+1)$-cells in the image of $\Omega$. We further set

$$
\operatorname{DFVol}_{k+1}^{c}(\Sigma):=\min \left\{\operatorname{vol}_{k+1}^{c}(\Omega) \mid \Omega \text { is an }(k+1) \text {-ball with boundary } \Sigma\right\} .
$$

The $k$-dimensional (combinatorial) Dehn function (of $K$ ) is then defined as

$$
\delta_{K}^{k}(l):=\max \left\{\operatorname{DFVol}_{k+1}^{c}(\Sigma) \mid \operatorname{vol}_{k}^{c}(\Sigma) \leq l\right\} .
$$

The above definition of higher dimensional Dehn functions is equivalent to that of Alonso et al. in [3] (see [13]). In particular its equivalence class (in the sense above) is independent of the chosen complex $K$ and defines a quasi-isometry invariant of $\Gamma$. 
We next give an alternative definition of higher-dimensional Dehn functions which is better adapted to our situation, since it is more closely related to filling functions.

Let $X$ be a $k$-connected manifold or Euclidean polyhedral complex and let $\Gamma$ be a (finitely generated) group which acts cocompactly on $X$. The $k$-th dimensional (Lipschitz) Dehn function (of $X$ ), $\delta_{X}^{k}$, is a homotopical version of a filling function. It measures the volume that is necessary to extend a Lipschitz map $f: S^{k} \rightarrow X$ of the $k$-dimensional unit sphere to a map $g: B^{k+1} \rightarrow X$ of the $(k+1)$-dimensional unit ball:

$$
\operatorname{DFVol}_{k+1}(f):=\inf \left\{\operatorname{vol}_{k+1}(g) \mid g: B^{k+1} \rightarrow X, g_{\left.\right|_{S}}=f\right\}
$$

and

$$
\delta_{X}^{k}(l):=\sup \left\{\operatorname{DFVol}_{k+1}(f) \mid f: S^{k} \rightarrow X, \operatorname{vol}_{k}(f) \leq l\right\} .
$$

As a consequence of the Federer-Fleming deformation theorem the combinatorial and Lipschitz versions of higher dimensional Dehn functions are actually equivalent if $X$ (or $K$ ) is a space which can be approximated by a polyhedral complex of bounded geometry (compare [1], Theorem 2.1.). Since its equivalence class defines a quasi-isometry invariant (by [3]), we will write $\delta_{\Gamma}^{k}$ in place of $\delta_{X}^{k}$ or $\delta_{K}^{k}$ to denote (a representative of) this equivalence class.

Theorem 1 implies

Theorem 2 (Dehn functions). Let $X=S \times E \times B$ be the metric product of a symmetric space of noncompact type $S=G / K$, a Euclidean space $E$ and a product $B$ of Euclidean buildings (one or two factors of $X$ may be trivial) and let $\operatorname{rank} X=$ $\operatorname{rank}_{\mathbb{R}} G+\operatorname{dim} E+\operatorname{dim} B$ denote the Euclidean rank of $X$.

Let $\Gamma$ be a group acting isometrically, cocompactly and properly discontinuously on X. Then

(i) the $k$-dimensional Dehn functions of $\Gamma$ are Euclidean below the rank:

$$
\delta_{\Gamma}^{k}(l) \sim l^{\frac{k+1}{k}} \text { if } k \leq \operatorname{rank} X-1 ;
$$

(ii) the $k$-dimensional Dehn functions of $\Gamma$ are linear above the rank:

$$
\delta_{\Gamma}^{k}(l) \sim l \quad \text { if } \operatorname{rank} X \leq k \leq \operatorname{dim} X-1 .
$$

As noted above, the chief examples of groups $\Gamma$ to which Theorem 2 applies are provided by cocompact S-arithmetic subgroups of reductive algebraic groups $\boldsymbol{G}$ which are defined over global fields $\mathbb{K}$. We will discuss such groups in some more detail in Section 4. Notice that, if $\mathbb{K}$ is a function field, then Theorem 2 reduces to part (i) only. Note further that in the special case where $X=S$ is a symmetric space of rank 1, a group $\Gamma$ as in Theorem 2 is hyperbolic and then Theorem 2 asserts that all Dehn functions are linear. For general hyperbolic groups linear upper bounds were obtained in [32] and [37]. 
As already mentioned, linear 1-dimensional Dehn functions characterize Gromovhyperbolic groups. On the other end of the range, top-dimensional linear isoperimetric inequalities (or, equivalently, positive Cheeger constants) characterize discrete nonamenable groups, which act geometrically (see [26], 05.A6, and Theorem 3 below).

Finally, we note that a theorem of Avez [4] asserts that the fundamental group of a closed manifold $V$ with nonpositive sectional curvature is non-amenable unless $V$ is flat. This in particular applies to fundamental groups of compact locally symmetric spaces (of noncompact type) with Euclidean factors, i.e., uniform lattices in real reductive Lie groups. Theorems 1 and 2 thus refine Avez's theorem in the case of reductive locally symmetric spaces.

Remark (Generalization). Theorem 1 and Theorem 2 extend to CAT(0) spaces (and groups acting geometrically on them) of the form $X=S \times H$, where $S$ is a symmetric space of noncompact type as before, and where $H$ is a Euclidean polyhedral complex with bounded geometry and the property that its dimension is equal to its Euclidean rank (i.e., the maximal dimension of an isometrically embedded Euclidean space). In fact, in the proofs below we only use these (weak) geometric properties of buildings.

\section{The proof of Theorem 1}

2.1. Theorem 1 (i): Euclidean fillings below the rank. By definition of the filling functions $\mathrm{FV}_{X}^{k+1}$ we have $k \geq 1$. As we are here concerned with Theorem 1 (i), we may assume that (Euclidean) rank $X \geq 2$.

Wenger proved Euclidean isoperimetric inequalities in a general setting:

Proposition 1 (Wenger, [47]). If $X$ is a complete simply connected CAT(0) space of dimension n, then any $k$-dimensional Lipschitz cycle $\Sigma$ in $X$ satisfies a Euclidean isoperimetric inequality:

$$
\mathrm{FVol}_{k+1}(\Sigma) \leq C_{k}\left(\operatorname{vol}_{k} \Sigma\right)^{\frac{k+1}{k}}, \quad 1 \leq k \leq n-1,
$$

where $C_{k}$ is a constant that depends only on $X$ and $k$.

These isoperimetric inequalities provide an upper bound for filling functions

$$
\mathrm{FV}_{X}^{k+1}(l) \prec l^{\frac{k+1}{k}} \text {. }
$$

In order to get also a lower bound for $k<r=\operatorname{rank} X$ we consider a $k$ dimensional round sphere $S$ of volume $l$ contained in a maximal ( $r$-dimensional) flat $F$ of $X$. Let $B$ be a minimal filling of $S$ in $X$. The orthogonal projection from $X$ to $F$ is a 1-Lipschitz map (see e.g. [14], 2.4). If we orthogonally project $B$ to $F$ we thus get a filling $B^{\prime}$ of $S$ in $F$ with $\operatorname{vol}_{k+1} B^{\prime} \leq \operatorname{vol}_{k+1} B$ (see [15], 5.2.2). Hence $B^{\prime}$ is a minimal filling of the round sphere $S$ in the Euclidean space $F$. By 
the solution of the isoperimetric problem in Euclidean space, $B^{\prime}$ must be a round ball with boundary $S$ and thus satisfies $l^{\frac{k+1}{k}} \sim \operatorname{vol}_{k+1} B^{\prime} \leq \operatorname{vol}_{k+1} B$. Hence we get the required lower bound

$$
l^{\frac{k+1}{k}} \prec \mathrm{FV}_{X}^{k+1}(l) .
$$

2.2. Theorem 1 (ii): Linear fillings above the rank. Note that Theorem 1 (ii), which concerns fillings of dimensions above $\operatorname{rank} X$, is only meaningful if the symmetric factor $S$ of $X$ is nontrivial. In fact, otherwise we have $X=E \times B$ and the (Euclidean) rank of $X$ coincides with the topological dimension $\operatorname{dim} X=\operatorname{dim} E+\operatorname{dim} B$ and hence there is no filling problem above the rank. In this subsection we will thus always assume that the symmetric factor $S$ is nontrivial.

2.2.1. Linear upper bounds. We first recall the formulae for Jacobi fields in Riemannian products $S \times M$, where $S=G / K$ is a symmetric space of noncompact type (with semisimple Lie group $G$ ) and $M$ is an open (flat) submanifold of some Euclidean space. These will be used below for volume estimates. A proof for semisimple symmetric spaces can be found in [19], 2.14. and 2.15; it directly extends to the product case. Recall that a Jacobi field along a geodesic ray is called stable, if its norm is bounded.

Proposition 2. Let $S \times M=G / K \times M$ be an $n$-dimensional Riemannian product of a symmetric space of noncompact type with an open (flat) submanifold $M \subset \mathbb{R}^{m}$ of a Euclidean space and let $x_{0}$ be a base point of $S$. Let $\mathfrak{g}=\mathfrak{k} \oplus \mathfrak{p}$ be a Cartan decomposition of the Lie algebra of the semisimple group $G$. Let a be a maximal abelian subspace of $\mathfrak{p}$. For a unit vector $H$ in $\mathfrak{a}$, an element $g \in G$ and a point $p \in M$, let

$$
c:[0, \infty) \rightarrow S \times M, \quad t \mapsto\left(g \cdot \exp t H \cdot x_{0}, p\right),
$$

be a geodesic ray parametrized by arc-length starting at $\left(g \cdot x_{0}, p\right)$. Further let $E_{l}(t), 0 \leq l \leq n-1$, be orthonormal parallel vector fields in $S \times M$ along the ray $c$ and orthogonal to $c$. Then any stable Jacobi field $Y(t)$ along $c(t)$ and orthogonal to $c$ with $Y(0)=\sum_{l=1}^{n-1} y_{l} E_{l}(0)$ is of the form

$$
Y(t)=\sum_{l=1}^{n-1} y_{l} e^{-\sqrt{\lambda_{l}} t} E_{l}(t),
$$

where the $\lambda_{l}$ are zero or eigenvalues of the curvature operator $R_{H}=(\operatorname{ad} H)_{\mid \mathfrak{p}}^{2}$ and thus either also equal to zero or of the form $\lambda_{l}=\left(\alpha_{l}(H)\right)^{2}$ for some (positive) root $\alpha_{l}$.

We now proceed to prove the linear upper bound in Theorem 1 (ii). To that end let $X=S \times E \times B$ be as in Theorem 1 (with nontrivial symmetric factor $S$ ). Further 
let $\Sigma$ be a $k$-dimensional cycle with

$$
k \geq r=\operatorname{rank} X=\operatorname{rank} S+\operatorname{dim} M=\operatorname{dim} a+\operatorname{dim} M .
$$

For convenience we use in the following the same notation for the map $\Sigma$ and its image in $X$. We wish to construct a chain $\Omega$ which bounds $\Sigma$ and such that $\operatorname{vol}_{k+1} \Omega \leq$ const $\cdot \operatorname{vol}_{k} \Sigma$, where the constant only depends on $X$ and $k$. The idea is to fill $\Sigma$ by first projecting it orthogonally to a suitable horosphere of $X$ and then take $\Omega$ as the cylinder of this map together with a filling of the projection (which will be very small, i.e., of volume $\leq 1$ ) inside this horosphere. We will achieve this in 6 steps.

Step 1 (A cone over $\Sigma$ ). Consider a Weyl chamber in the geometric boundary of the symmetric factor $S=G / K$ and denote its barycenter by $\beta$. Together with the base point $x_{0}$ of $S$ this defines an Iwasawa decomposition $G=N A K$ and associated horocyclic coordinates $S \cong N A \cdot x_{0}$ (see e.g. [33]). Let $c_{1}(t):=\exp t H_{0} \cdot x_{0}, t \in \mathbb{R}$, be the unit speed geodesic in $S$ through $x_{0}$ and asymptotic to $\beta$. Note that taking translates of $c_{1}$ gives a foliation of $S$ by asymptotic geodesics: $S=N \cdot \exp H_{0}^{\perp}$. $c_{1}(\mathbb{R})$.

Given a point $x=\left(n \exp H_{1} c_{1}(s), e, b\right) \in X=S \times E \times B$, with $H_{1} \in H_{0}^{\perp}$, we denote by $\tau(x, t)$ the unique geodesic ray in $X$ which starts at $x$ and is asymptotic to the above barycenter $\beta \in S(\infty) \subset X(\infty)$, i.e.,

$$
\tau(x, t)=\left(n \exp H_{1} \cdot c_{1}(s+t), e, b\right), \quad t \in[0, \infty) .
$$

This in particular defines a map

$$
\tau: \Sigma \times \mathbb{R}_{\geq 0} \rightarrow X, \quad(x, t) \mapsto \tau(x, t) .
$$

Below we will use the associated $(k+1)$-dimensional "cone" $\widetilde{\Omega}:=\tau\left(\Sigma \times \mathbb{R}_{\geq 0}\right) \subset X$ over the $k$-cylce $\Sigma$.

In order to guarantee that $\widetilde{\Omega}$ is $(k+1)$-dimensional, we pick some point $\tilde{x} \in \Sigma$ and choose the barycenter $\beta$ in such a way that the geodesic ray $\tau(\tilde{x}, t)$ is transversal to $\Sigma$. That this is possible for any $k$ follows e.g. from the polar decomposition of $S$ (and its boundary at infinity): [30], Chapter 5, Lemma 6.3. We also note that the image under $\tau$ of the set of those points of $\Sigma$ where the issuing geodesic ray is tangential (i.e. not transversal) is only a $k$-dimensional subset and hence does not contribute to the $(k+1)$-volume of the cone $\widetilde{\Omega}$.

Step 2 (Regular points of $\Sigma$ ). If the building factor $B$ of $X$ is nontrivial, $X$ is not a differentiable manifold. We can, however, consider all open cells of $B$, say $\sigma_{m}$ with $m$ in some index set $I_{B}$. Then there is a finite subset $I \subset I_{B}$ such that the intersections

$$
\Sigma_{m}:=\Sigma \cap\left(S \times E \times \sigma_{m}\right), \quad m \in I,
$$

have positive $k$-dimensional Hausdorff measure and cover $\Sigma$ up to a set of measure zero (recall that we use here the same notation for the map $\Sigma$ and its image in $X$ ). 
Notice that $S \times E \times \sigma_{m}, m \in I_{B}$, is the Riemannian product of a symmetric space with an (open, flat) Euclidean manifold, so that Proposition 2 can be applied.

We fix an $\epsilon>0$, which will be chosen appropriately in Step 5. By Rademacher's theorem $\Sigma_{m}$ is differentiable almost everywhere (with respect to Hausdorff measure). By Lusin's theorem (see [21], 2.3.5) there is a compact subset $\Sigma_{m}^{r}$ of $\Sigma_{m}$ for each $m \in I$, such that $\Sigma_{m}^{r}$ is a $C^{1}$-manifold and $\operatorname{vol}_{k}\left(\Sigma_{m} \backslash \Sigma_{m}^{r}\right)<\epsilon$.

The sets $\Sigma_{m}^{r}$ are non-empty, disjoint $C^{1}$-manifolds which cover the regular part

$$
\Sigma^{r}:=\bigcup_{m \in I} \Sigma_{m}^{r}
$$

of $\Sigma$. Note that $\operatorname{vol}_{k}\left(\Sigma \backslash \Sigma^{r}\right)<\epsilon|I|$.

Step 3 (Volume of the cone over the regular part $\Sigma^{r}$ ). We fix $m \in I$ and, for $\tau$ as ins Step 1 , we denote by $\omega$ be the volume form on $\widetilde{\Omega}_{m}^{r}:=\tau\left(\Sigma_{m}^{r} \times \mathbb{R}_{\geq 0}\right) \subset X$. We also define $\phi: \Sigma_{m}^{r} \times \mathbb{R}_{\geq 0} \rightarrow \mathbb{R}$ by

$$
\tau^{*} \omega(x, t)=\phi(x, t) d t \wedge \pi^{*} \bar{\omega}(x)
$$

where $\pi: \Sigma_{m}^{r} \times \mathbb{R}_{\geq 0} \rightarrow \Sigma_{m}^{r}$ is the projection to the first factor and $\bar{\omega}$ is the volume form on $\Sigma_{m}^{r}$. We will show that there is a constant $C_{2}$ depending only on $X$ such that

$$
\operatorname{vol}_{k+1}\left(\widetilde{\Omega}_{m}^{r}\right) \leq C_{2} \operatorname{vol}_{k}\left(\Sigma_{m}^{r}\right) \quad \text { for all } m \in I \text {. }
$$

Equation (2) in turn implies the following linear estimate for the cone over the regular part $\Sigma^{r}$ of $\Sigma$

$$
\operatorname{vol}_{k+1}\left(\tilde{\Omega}^{r}\right)=\sum_{m \in I} \operatorname{vol}_{k+1}\left(\widetilde{\Omega}_{m}^{r}\right) \leq C_{2} \sum_{m \in I} \operatorname{vol}_{k}\left(\Sigma_{m}^{r}\right)=C_{2} \operatorname{vol}_{k}\left(\Sigma^{r}\right) .
$$

In order to prove estimate (2) we will use the following

Lemma 1. Let $k \geq \operatorname{rank} X, m \in I$ and $\epsilon$ as above. Then there are constants $\lambda_{*}>0$, $C_{1}>0$ and $C_{2}>0$ depending only on $X$ (but not on $k, m$ or $\epsilon$ ) such that for every $x \in \Sigma_{m}^{r}$ the density function $\phi$ in (1) satisfies

$$
\phi(x, t) \leq C_{1} e^{-\sqrt{\lambda_{*}} t} \quad \text { and thus } \quad \int_{0}^{\infty} \phi(x, t) d t=C_{2}<\infty .
$$

Proof. Consider the geodesic ray $c(t):=\tau(x, t)$ defined in Step 1 starting at $x \in \Sigma_{m}^{r}$ and asymptotic to $\beta$. We can (and do) assume that $c$ is transversal to $\Sigma$ (see the discussion in Step 1). Let $V_{0}(0), \ldots, V_{n-1}(0)$ be an orthonormal frame of $T_{x} X$ such that $V_{0}(0)=\dot{c}(0)$ and $V_{0}(0), \ldots, V_{k}(0)$ span $T_{x} \widetilde{\Omega}_{m}^{r}$. For each $0 \leq i \leq k$ the unit vector $V_{i}(0)$ extends to a parallel vector field $V_{i}(t)$ along $c(t)$. We choose local coordinates $\left(x_{1}, \ldots, x_{k}\right)$ in $\Sigma_{m}^{r}$ around $x$ such that $\frac{\partial}{\partial x_{i}}(x)=V_{i}(0)+a_{i} V_{0}(0)$. Then, 
by the map $\tau$, we have local coordinates $\left(x_{1}, \ldots, x_{k}, t\right)$ in $\widetilde{\Omega}_{m}^{r}$ near the geodesic ray $c(t)$. The volume form $\bar{\omega}$ of $\Sigma_{m}^{r}$ at $x$ is

$$
\bar{\omega}(x)=\sqrt{1+\sum_{i} a_{i}^{2}} d x_{1} \wedge \cdots \wedge d x_{k} .
$$

We now consider the (unique) stable Jacobi fields $Y_{i}(t)$ along $c(t)$ which satisfy $Y_{i}(0)=V_{i}(0), 1 \leq i \leq k$. Using the $k \times k$ matrix

$$
A_{k}(t):=\left(\left\langle Y_{i}(t), Y_{j}(t)\right\rangle\right)_{1 \leq i, j \leq k}
$$

we can write the volume element $\omega$ on $\widetilde{\Omega}_{m}^{r}$ as

$$
\omega(c(t))=\sqrt{\operatorname{det} A_{k}(t)} d t \wedge d x_{1} \wedge \cdots \wedge d x_{k} .
$$

Hence we get

$$
\phi(x, t)=\sqrt{\frac{\operatorname{det} A_{k}(t)}{1+\sum_{i} a_{i}^{2}}} \leq \sqrt{\operatorname{det} A_{k}(t)} .
$$

We now choose orthonormal parallel vector fields $E_{l}(t), 0 \leq l \leq n-1$, along the ray $c(t)$ as in Proposition 2 such that, for $1 \leq i \leq n-1$,

$$
V_{i}(0)=\sum_{l=1}^{n-1} a_{i l} E_{l}(0) .
$$

Note that the matrix $\left(a_{i l}\right)$ is orthogonal. By Proposition 2, the Jacobi fields $Y_{i}$, $1 \leq i \leq k$, can be written as

$$
Y_{i}(t)=\sum_{l=1}^{n-1} e^{-\sqrt{\lambda_{l}} t} a_{i l} E_{l}(t) .
$$

Hence

$$
\begin{aligned}
\left\langle Y_{i}(t), Y_{j}(t)\right\rangle & =\left\langle\sum_{l=1}^{n-1} e^{-\sqrt{\lambda_{l}} t} a_{i l} E_{l}(t), \sum_{m=1}^{n-1} e^{-\sqrt{\lambda_{m}} t} a_{j m} E_{m}(t)\right\rangle \\
& =\sum_{l=1}^{n-1} e^{-2 \sqrt{\lambda_{l}} t} a_{i l} a_{j l} .
\end{aligned}
$$

In order to estimate the determinant of the matrix

$$
A_{k}(t):=\left(\left\langle Y_{i}(t), Y_{j}(t)\right\rangle\right)_{1 \leq i, j \leq k}
$$

we consider the $k \times(n-1)$ matrices

$$
A:=\left(a_{i l}\right) \quad \text { and } \quad B:=\left(a_{j l} e^{-2 \sqrt{\lambda_{l}} t}\right) .
$$


Then, by the Binet-Cauchy formula (see e.g. [22]), we have

$$
\operatorname{det} A_{k}(t)=\operatorname{det}\left(A B^{\top}\right)=\sum_{1 \leq s_{1}<s_{2}<\cdots<s_{k} \leq n-1} \operatorname{det} A^{s_{1} \ldots s_{k}} \operatorname{det} B^{s_{1} \ldots s_{k}},
$$

where $A^{s_{1} \ldots s_{k}}$ denotes the $k \times k$ matrix consisting of the columns of $A$ with indices $s_{1} \ldots s_{k}$ and similarly for $B$. By definition $\left(a_{i j}\right)_{1 \leq i, j \leq n-1}$ is an orthogonal matrix and hence $\left|a_{i j}\right| \leq 1$. By Proposition 1, the $\lambda_{i}$ are either zero or eigenvalues of the curvature operator given by $H_{0}$. By assumption we have $k \geq \operatorname{rank} X$. Since the projection of the geodesics $\tau(x, t)$ to $S$ are regular by definition, at most $k-1$ of the Jacobi fields $Y_{i}$ can be tangent to a flat in $X$. Thus, for each matrix $B^{s_{1} \ldots s_{k}}$, there is at least one column $s_{i}$, say, such that $\lambda_{s_{i}}=\alpha_{s_{i}}^{2}\left(H_{0}\right)>0$ for some positive root $\alpha_{s_{i}}$. Moreover, by the choice of $H_{0}$ as a barycentric direction, these values are uniformly bounded away from zero by $\lambda_{*}:=\min _{\alpha \in \Sigma^{+}} \alpha\left(H_{0}\right)>0$. We thus obtain the estimate

$$
\operatorname{det} A_{k}(t) \leq C_{1}^{2} e^{-2 \sqrt{\lambda_{*}} t},
$$

where $C_{1}^{2}$ depends only on $n$. Inserting (5) in (4) completes the proof of Lemma 1.

From Lemma 1 we immediately get (2):

$$
\operatorname{vol}_{k+1}\left(\widetilde{\Omega}_{m}^{r}\right)=\int_{\Sigma_{m}^{r}}\left(\int_{0}^{\infty} \phi(x, t) d t\right) \bar{\omega}(x)=C_{2} \operatorname{vol}_{k}\left(\Sigma_{m}^{r}\right) .
$$

Step 4 (Volume of the cone over the singular part $\Sigma \backslash \Sigma^{r}$ ). We claim that for $a \in[0, \infty$ ) holds

$$
\operatorname{vol}_{k+1}\left(\tau\left(\Sigma \backslash \Sigma^{r} \times[0, a]\right)\right) \leq 3^{k+1} \operatorname{vol}_{k}\left(\Sigma \backslash \Sigma^{r}\right) \cdot a .
$$

In fact, this immediately follows from

Lemma 2. The map $\tau: \Sigma \times[0, \infty) \rightarrow X$ is 3-Lipschitz.

Proof. Let $x, y \in \Sigma$. By construction the geodesics $\tau(x, t)$ and $\tau(y, t)$ are asymptotic. Thus by the convexity of the distance function in the CAT( $(0)$ space $X$ we have for $s, t \geq 0$

$$
\begin{aligned}
d_{X}(\tau(x, s), \tau(y, t)) & \leq d_{X}(\tau(x, s), \tau(y, s))+d_{X}(\tau(y, s), \tau(y, t)) \\
& \leq d_{X}(\tau(x, 0), \tau(y, 0))+|s-t| \\
& =d_{X}(x, y)+|s-t| \\
& \leq 3 \sqrt{d_{X}(x, y)^{2}+|s-t|^{2}} .
\end{aligned}
$$

Step 5 (A chain $\Omega$ which fills $\Sigma$ ). By Lemma 1 and since $I$ is finite we can find $t_{0} \geq 1$ so large that $\phi\left(x, t_{0}\right) \leq\left(|I| \operatorname{vol}_{k}\left(\Sigma_{m}^{r}\right)\right)^{-1}$ for all $m \in I$ (note that $I$ depends on $\Sigma$ ). 
Consider a horosphere $\mathscr{H}$ based at $\beta \in X(\infty)$ (as in Step 1) such that $\Sigma$ lies in the complement of the horoball $B_{\mathscr{H}}$ with boundary $\mathscr{H}$. Let $\pi_{\mathscr{H}}: X \rightarrow \mathscr{H}$ denote the horospherical projection. The image $\pi_{\mathscr{H}}(\Sigma)$ can be written in the form

$$
\pi_{\mathscr{H}}(\Sigma)=\{\tau(x, t(x)) \mid x \in \Sigma\} .
$$

We choose $\mathscr{H}$ in such a way that $t(x) \geq t_{0}$ for all $x \in \Sigma$.

By formula (1) and the choice of $t_{0}$ we the get

$$
\begin{aligned}
\operatorname{vol}_{k}\left(\pi_{\mathscr{H}}\left(\Sigma^{r}\right)\right) & =\sum_{m \in I} \operatorname{vol}_{k}\left(\pi_{\mathscr{H}}\left(\Sigma_{m}^{r}\right)\right) \\
& =\sum_{m \in I} \int_{\Sigma_{m}^{r}} \phi(x, t(x)) \bar{\omega}(x) \\
& \leq \sum_{m \in I} \int_{\Sigma_{m}^{r}} \phi\left(x, t_{0}\right) \bar{\omega}(x) \\
& \leq \sum_{m \in I}\left(|I| \operatorname{vol}_{k}\left(\Sigma_{m}^{r}\right)\right)^{-1} \operatorname{vol}_{k}\left(\Sigma_{m}^{r}\right)=1 .
\end{aligned}
$$

For the horosphere $\mathscr{H}$ chosen above we set $t_{1}:=\max \{t(x) \mid x \in \Sigma\}$ (we can assume that $\left.t_{1} \geq 1\right)$. We now also choose the parameter $\epsilon$ of Step 2 explicitly as $\epsilon:=\left(t_{1}|I|\right)^{-1}$.

Notice that the horospherical projection $\pi_{\mathscr{H}}$ is 1-Lipschitz. By the definition of $\epsilon$ in Step 2 and the explicit choice above we thus have

$$
\operatorname{vol}_{k}\left(\pi_{\mathscr{H}}\left(\Sigma \backslash \Sigma^{r}\right)\right) \leq \operatorname{vol}_{k}\left(\Sigma \backslash \Sigma^{r}\right)<\epsilon|I|=\frac{1}{t_{1}} \leq 1 .
$$

We conclude that

$$
\operatorname{vol}_{k}\left(\pi_{\mathscr{H}}(\Sigma)\right) \leq 1+1=2 .
$$

As this is independent of $\Sigma$ and since $X$ has bounded geometry, there is a filling $\Omega_{0}$ of $\pi_{\mathscr{H}}(\Sigma)$ such that

$$
\operatorname{vol}_{k+1}\left(\Omega_{0}\right) \leq C_{3},
$$

where $C_{3}$ is again a uniform constant depending only on $X$. Finally, if $B_{\mathscr{H}}$ denotes the horoball in $X$ with boundary $\mathscr{H}$, we set

$$
\Omega:=\left(\widetilde{\Omega} \backslash B_{\mathscr{H}}\right) \cup \Omega_{0}=\left(\tau\left(\Sigma \times \mathbb{R}_{\geq 0}\right) \backslash B_{\mathscr{H}}\right) \cup \Omega_{0} .
$$

By construction this $\Omega$ is a filling $(k+1)$-chain for $\Sigma$.

Step 6 (Volume of $\Omega$ ). Let $t_{0}, t_{1}$ and $\epsilon=\left(t_{1}|I|\right)^{-1}$ be as in Step 5. From Step 4 we have

$$
\begin{aligned}
\operatorname{vol}_{k+1} & \left(\tau\left(\Sigma \backslash \Sigma^{r} \times[0, \infty)\right) \backslash B \mathscr{H}\right) \\
& \leq \operatorname{vol}_{k+1}\left(\tau\left(\Sigma \backslash \Sigma^{r} \times\left[0, t_{1}\right]\right)\right) \\
& \leq 3^{k+1} \operatorname{vol}_{k}\left(\Sigma \backslash \Sigma^{r}\right) \cdot t_{1} \leq 3^{k+1} \epsilon|I| t_{1} \\
& =3^{k+1}=: C_{4} .
\end{aligned}
$$


This together with (3) and (6) yields

$$
\begin{aligned}
\operatorname{vol}_{k+1}(\Omega)= & \operatorname{vol}_{k+1}\left(\tau\left(\Sigma^{r} \times[0, \infty)\right) \backslash B \mathscr{H}\right) \\
& +\operatorname{vol}_{k+1}\left(\tau\left(\Sigma \backslash \Sigma^{r} \times[0, \infty)\right) \backslash B_{\mathscr{H}}\right)+\operatorname{vol}_{k+1}\left(\Omega_{0}\right) \\
\leq & \operatorname{vol}_{k+1}\left(\tilde{\Omega}^{r}\right)+C_{4}+C_{3} \\
\leq & C_{2} \operatorname{vol}_{k}\left(\Sigma^{r}\right)+C_{4}+C_{3} \\
\leq & C_{2} \operatorname{vol}_{k}(\Sigma)+C_{3}+C_{4} \\
\prec & \operatorname{vol}_{k}(\Sigma) .
\end{aligned}
$$

This estimate completes the proof of the linear upper bound in Theorem 1 (ii).

2.2.2. Linear lower bounds. In order to establish linear lower bounds for the filling functions in Theorem 1 (ii), it suffices to provide arbitrarily large $k$-cycles $\Sigma$, with the property that any $(k+1)$-filling $\Omega$ satisfies $\operatorname{vol}_{k+1}(\Omega) \succ \operatorname{vol}_{k}(\Sigma)$. We construct such cycles via the following lemma. Recall that every symmetric space $S$ of noncompact type contains (many) copies of real hyperbolic planes $\mathbb{H}^{2}$ (see [30], IX.2).

Lemma 3. Let $S$ be an $n$-dimensional symmetric space of noncompact type with base point $x_{0}$. Let $\mathbb{H}^{2} \subset S$ be a totally geodesic hyperbolic plane containing $x_{0}$. For any $2 \leq k \leq n$ there exists a $(k-2)$-dimensional subspace $q$ of $\mathfrak{p} \cong T_{x_{0}} S$ orthogonal to $\mathfrak{h} \cong T_{x_{0}} \mathbb{H}^{2}$ such that the $k$-dimensional submanifold

$$
W:=\exp (\mathfrak{h} \oplus \mathfrak{q}) \cdot x_{0}
$$

of $S$, which is diffeomorphic to $\mathbb{H}^{2} \times \exp q \cdot x_{0}=: \mathbb{H}^{2} \times Q$, has the following property:

Let $\Sigma$ be the topological sphere obtained as the intersection of $W$ with the sphere $S_{R}\left(x_{0}\right)$ of radius $R>>1$ and center $x_{0}$ in $S$. Then the $k$-dimensional Hausdorff measure (or Riemannian volume) of any (smooth) filling $\Omega$ of $\Sigma$ in $S$ satisfies

$$
\operatorname{vol}_{k}(\Omega) \succ e^{R} \succ \operatorname{vol}_{k-1}(\Sigma) .
$$

Proof. The proof is by induction on the dimension of $W$ and uses the coarea formula.

We start the induction with $k=2$. In this case we take $\mathfrak{q}:=\{0\}$, i.e., $W=\mathbb{H}^{2}$. Consider the circle $\Sigma_{1}:=S_{R}\left(x_{0}\right) \cap W \subset W$. Fill $\Sigma_{1}$ with any 2-chain $\Omega_{2}$ in $S$. The orthogonal projection of $S$ to the totally geodesic submanifold $W=\mathbb{H}^{2}$ is 1-Lipschitz. Hence, if $\Omega_{2}^{\prime}$ is the orthogonal projection of $\Omega_{2}$ to $W$, we have $\operatorname{vol}_{2}\left(\Omega_{2}\right) \geq \operatorname{vol}_{2}\left(\Omega_{2}^{\prime}\right)$, i.e, the filling $\Omega_{2}^{\prime}$ of $\Sigma_{1}$ in $W=\mathbb{H}^{2}$ is even smaller than the filling in $S$. The minimal filling of the circle $\Sigma_{1}$ in $W=\mathbb{H}^{2}$ is the disc $D_{R}^{2}$ of radius $R$. Hence

$$
\operatorname{vol}_{2}\left(\Omega_{2}^{\prime}\right) \geq \operatorname{vol}_{2}\left(D_{R}^{2}\right) \sim e^{R} \sim \operatorname{vol}_{1}\left(\Sigma_{1}\right)
$$

and the claim follows in this case.

In order to continue the proof by induction, we assume that the lemma is proved for suitable submanifolds $W^{2}, W^{3}, \ldots, W^{k}$ with $\operatorname{dim} W^{i}=i$. We now wish to 
prove the lemma for a $W^{k+1}$ of dimension $k+1$. To that end we choose a geodesic $c$ in $S$ orthogonal to $W^{k}$ and define $W^{k+1}$ as the union of the parallel translates of $W^{k}$ along $c$. Let $\pi: S \rightarrow c$ denote the orthogonal projection of $S$ to the geodesic $c$. Since $\pi$ is 1-Lipschitz, Eilenberg's coarea formula (see [16], 13.3) yields for any filling $\Omega_{k+1}$ of $\Sigma_{k}:=S_{R}\left(x_{0}\right) \cap W^{k+1}$ in $S$

$$
\begin{aligned}
\operatorname{vol}_{k+1}\left(\Omega_{k+1}\right) & \succ \int_{-\infty}^{\infty} \operatorname{vol}_{k}\left(\pi^{-1}(t) \cap \Omega_{k+1}\right) d t \\
& \geq \int_{-1}^{+1} \operatorname{vol}_{k}\left(\pi^{-1}(t) \cap \Omega_{k+1}\right) d t .
\end{aligned}
$$

The last integral is greater than the minimal filling volume of the intersection of $S_{R}\left(x_{0}\right)$ with the "thickening" of $W^{k}=\pi^{-1}(0) \cap W^{k+1}$ given by

$$
\bigcup_{t \in(-1,+1)} \pi^{-1}(t) \cap W^{k+1} \text {. }
$$

The latter consists of copies of $W^{k}$ (note that $\pi$ commutes with parallel translation along $c$ ).

By Sard's theorem, almost every $t \in[-1,1]$ is a regular value of $\pi \mid \Omega_{k+1}$ and $\pi \mid \Sigma_{k}$. Hence

$$
\partial\left(\pi^{-1}(t) \cap \Omega_{k+1}\right)=\pi^{-1}(t) \cap \partial \Omega_{k+1}=\pi^{-1}(t) \cap \Sigma_{k},
$$

i.e., $\pi^{-1}(t) \cap \Omega_{k+1}$ is a filling of $\pi^{-1}(t) \cap \Sigma_{k}$ for a.e. $t \in[-1,1]$ (see e.g. 2.1. in [28]). Now we have

$$
\pi^{-1}(0) \cap \Sigma_{k}=\pi^{-1}(0) \cap W^{k+1} \cap S_{R}\left(x_{0}\right)=W^{k} \cap S_{R}\left(x_{0}\right)=\Sigma_{k-1} .
$$

We thus can apply the induction hypothesis and get that $\pi^{-1}(0) \cap \Omega_{k+1}$ and hence also $\pi^{-1}(t) \cap \Omega_{k+1}$ for $t \in\left[-\frac{1}{100}, \frac{1}{100}\right]$, say, has Hausdorff measure (or Riemannian volume)

$$
\operatorname{vol}_{k}\left(\pi^{-1}(t) \cap \Omega_{k+1}\right) \succ e^{R} .
$$

Inequality (7) then yields $\operatorname{vol}_{k+1}\left(\Omega_{k+1}\right) \succ e^{R}$.

Finally, we need to show that $\operatorname{vol}_{k}\left(\Sigma_{k}\right) \prec e^{R}$. But $\Sigma_{k}$ is a sphere of radius $R$ in $W^{k+1}$ with center $x_{0}$ and $W^{k+1}$ contains a hyperbolic plane through $x_{0}$. The claim then follows from the computation of volumes of spheres via Jacobi fields (see [42], II.5, Lemma 5.4). This finishes the induction proof of Lemma 2.

We proceed to prove the lower bounds in Theorem 1 (ii). Let $\Sigma_{k}$ be a $k$-sphere of radius $R$ in $S \subset X=S \times E \times B$ as in Lemma 2 and let $\Omega_{k+1}$ be an arbitrary filling of $\Sigma_{k}$ in $X$. Let $\pi: X \rightarrow S$ be the orthogonal projection. As $S$ is a convex subspace of $X$ this again is a 1-Lipschitz map. Hence, by Lemma 2,

$$
\operatorname{vol}_{k+1}\left(\Omega_{k+1}\right) \geq \operatorname{vol}_{k+1}\left(\pi\left(\Omega_{k+1}\right)\right) \succ \operatorname{vol}_{k}\left(\Sigma_{k}\right) .
$$


In order to get $k$-cycles having at least linear fillings for any chain in $X$ for $k$ up to $\operatorname{dim} X-1=\operatorname{dim} S+\operatorname{dim} E+\operatorname{dim} B-1$ we choose a flat $F$ in $E \times B$ and consider the convex subspace $S \times F$ in $X$. Note that this actually is a manifold of dimension equal to $\operatorname{dim} X$. For the construction of the $\Sigma_{k}$ with $k \geq \operatorname{dim} S$ we then proceed exactly as in the last step of the proof of the Lemma 2 by inductively defining suitable $W^{k+1} \subset S \times F \subset X$ using successively parallel translation along and orthogonal projection to pairwise orthogonal geodesics in $F$. Finally, since the orthogonal projection from $X$ to $S \times F$ is 1-Lipschitz, we get the linear filling inequality by the same argument as above.

\section{The proof of Theorem 2}

We have the following general result relating filling and Dehn functions:

Proposition 3. Let $X_{1}$ and $X_{2}$ be two $k$-connected manifolds or simplicial complexes on which a group $\Gamma$ acts geometrically, then

$$
\delta_{X_{1}}^{k} \sim \delta_{X_{2}}^{k} \text { and } \mathrm{FV}_{X_{1}}^{k+1} \sim \mathrm{FV}_{X_{2}}^{k+1} .
$$

Moreover $\delta_{X_{1}}^{2} \prec \mathrm{FV}_{X_{1}}^{3}$ and $\delta_{X_{1}}^{k} \sim \mathrm{FV}_{X_{1}}^{k+1}$ for $k \geq 3$.

Proof. The first assertion is proved in [3], Section 6, Corollary 3, for a (simplicial) version of $\delta^{k}$. This is equivalent to the above (compare [49]). The second claim follows from [25], 2. $\mathrm{A}^{\prime}$, for upper bounds and [11], 2.6. (4), for lower bounds.

We emphasize that Young constructed examples of groups $\Gamma$ acting geometrically on a space $X$ for which $\delta_{\Gamma}^{2} \not \mathrm{FV}_{X}^{3}$ (see [49], Corollary 6). If, however, $X=S \times E \times B$ is as in Theorem 1, then we also have $\delta_{X}^{2} \succ \mathrm{FV}_{X}^{3}$. In fact, by Theorem 1 and the explicit construction of lower bounds by filling spheres by balls in Sections 2.1 and 2.2.2 we have

$$
\begin{array}{ll}
\delta_{X}^{2}(l) \succ l^{\frac{3}{2}} \sim \mathrm{FV}_{X}^{3}(l) & \text { if } \operatorname{rank} X \geq 3, \\
\delta_{X}^{2}(l) \succ l \sim \mathrm{FV}_{X}^{3}(l) & \text { if } \operatorname{rank} X=1 \text { or } 2 .
\end{array}
$$

Hence in that case there are no cycles that are "harder to fill" than spheres. Together with this observation and the fact (by [3]) that $\delta_{\Gamma}^{k}$ is a quasi-isometry invariant, i.e., $\delta_{\Gamma}^{k} \sim \delta_{X}^{k}$, Theorem 2 follows immediately from Theorem 1 and Proposition 3.

\section{Basic examples}

Examples of groups $\Gamma$ to which Theorem 2 applies include: 
(1) Crystallographic groups, i.e., discrete groups of isometries acting cocompactly on some Euclidean space $\mathbb{E}^{n}$ (see e.g. [46], Part II, Chapter 3).

(2) Uniform lattices in semisimple real Lie groups $G$, which act cocompactly on the associated symmetric space $S=G / K$ of noncompact type (see e.g. [7]).

(3) Uniform $S$-arithmetic groups, which act cocompactly on products of symmetric spaces of noncompact type and of Bruhat-Tits buildings. We briefly describe these last examples and some of their properties in the next paragraph.

4.1. S-arithmetic groups. We briefly recall the definition of $S$-arithmetic groups. For more details and informations we refer to [9] and [36], Chapter I.3.

A global field $\mathbb{K}$ is either an algebraic number field, i.e., a finite extension of $\mathbb{Q}$, or the function field of an algebraic curve over a finite field, i.e., a finite extension of $\mathbb{F}_{q}(T)$, the field of rational functions in one variable over the finite field with $q$ elements. In the first case the characteristic of $\mathbb{K}$ is zero and in the second it is positive. Completions of global fields with respect to archimedean or non-archimedean valuations are local fields.

Let $\mathbb{K}$ be a global field and let $S$ be a finite set of inequivalent valuations of $\mathbb{K}$ including all archimedean ones. The ring of $S$-integers of $\mathbb{K}$ is defined as

$$
\mathcal{O}_{S}:=\left\{\left.k \in \mathbb{K}|| k\right|_{v} \leq 1 \text { for all } v \notin S\right\}
$$

Let $\boldsymbol{G} \subset \boldsymbol{G} \boldsymbol{L}_{N}$ be a connected, reductive $\mathbb{K}$-group. The associated S-arithmetic group is the group of $S$-integers, $\boldsymbol{G}\left(\mathcal{O}_{S}\right)=\boldsymbol{G} \cap \boldsymbol{G} \boldsymbol{L}_{N}\left(\mathcal{O}_{S}\right)$. Its diagonal embedding into the locally compact group $\boldsymbol{G}_{S}:=\prod_{v \in S} \boldsymbol{G}\left(\mathbb{K}_{v}\right)$ (here $\mathbb{K}_{v}$ is the completion of $\mathbb{K}$ with respect to $v$ ) is an irreducible lattice. The reductive group $\boldsymbol{G}_{S}$ in turn acts isometrically on an associated CAT( 0$)$ space $X$ as in Theorem 1 or 2, i.e., the metric product of a symmetric space of noncompact type, a Euclidean space and a product of Euclidean (Bruhat-Tits) buildings.

4.2. Some (non-)existence results. We list a few results concerning existence and non-existence of uniform $S$-arithmetic groups.

- (Borel-Harder, [14]) Let $\boldsymbol{G}$ be a reductive $\mathbb{L}$-group over a local field $\mathbb{L}$ of characteristic zero. Then the group of $\mathbb{L}$-points, $\boldsymbol{G}(\mathbb{L})$, contains uniform lattices.

- (Borel-Harish-Chandra, [8]; see also [36], I.3.2.7) Let $\mathbb{K}$ be a global field. If $\boldsymbol{G}$ is $\mathbb{K}$-anisotropic and if one chooses the finite set $S$ in such a way that $\boldsymbol{G}$ is $\mathbb{K}_{v}$-isotropic for any $v \in S$, then (the diagonal embedding of) $\boldsymbol{G}\left(\mathcal{O}_{S}\right)$ is a uniform lattice in $\boldsymbol{G}_{S}$ and thus acts cocompactly on $X$. Note that many such $S$ exist, since the set of valuations $v$ such that $G$ is anisotropic over $\mathbb{K}_{v}$ is finite (see [44], Lemma 4.9).

- (Harder, [29], 3. Korollar 1) Only groups of type $\mathbb{A}$ have anisotropic forms over global fields of positive characteristic. In particular, if at least one of the 
factors $\boldsymbol{G}\left(\mathbb{K}_{v}\right)$ in $\boldsymbol{G}_{S}$ has char $\mathbb{K}_{v}>0$ and is not of type $\mathbb{A}$, then $\boldsymbol{G}_{S}$ cannot contain uniform lattices.

- If $\mathbb{K}$ is a global field and $\boldsymbol{G}$ is $\mathbb{K}$-isotropic, then $\boldsymbol{G}\left(\mathcal{O}_{S}\right)$ is a non-uniform lattice.

4.3. Finiteness properties. The very definition of higher-dimensional Dehn functions for some group $\Gamma$ requires that $\Gamma$ acts on highly connected spaces: $k$-dimensional Dehn functions are meaningful only for groups of finiteness type $F_{k+1}$.

In the case of number fields we have the following result:

(Raghunathan [40], Borel-Serre [9]) Any $S$-arithmetic subgroup $\boldsymbol{G}\left(\mathcal{O}_{S}\right)$ (uniform or not) of a reductive group $\boldsymbol{G}$ defined over a global number field is of finiteness type $F_{\infty}$

In the case of function fields there are restrictions:

(Serre [43], Kropholler-Mislin [31], Bux-Wortman [17]) An S-arithmetic subgroup $\boldsymbol{G}\left(\mathcal{O}_{S}\right)$ of a reductive group $\boldsymbol{G}$ defined over a function field $\mathbb{K}$ is of type

$F_{\infty}$ if and only if $\boldsymbol{G}$ is $\mathbb{K}$-anisotropic (or, equivalently, if $\boldsymbol{G}\left(\mathcal{O}_{S}\right)$ is uniform).

Hence for uniform $S$-arithmetic groups Dehn functions are always defined in any dimension. By the above result of Borel and Serre this also holds for non-uniform $S$-arithmetic groups in the number field case. We emphasize however that this is no longer true for non-uniform $S$-arithmetic subgroups of $\mathbb{K}$-isotropic groups $\boldsymbol{G}$ defined over function fields. Recall that the finiteness length of a group $\Gamma$ is the maximal $m$ such that $\Gamma$ has finiteness type $F_{m}$. In [17] and [18] it is proved that if $\boldsymbol{G}\left(\mathcal{O}_{S}\right)$ is non-uniform (and $\boldsymbol{G}$ defined over a function field), then its finiteness length is $r-1$, where $r$ is the sum of the local ranks (or, equivalently, the Euclidean rank of the associated product of Euclidean buildings on which $\boldsymbol{G}\left(\mathcal{O}_{S}\right)$ acts). In particular, if $k \geq r-1$, the the $k$-dimensional Dehn function is not defined for such groups. We address a conjectural picture about Dehn functions for non-uniform lattices in the next section.

\section{Some conjectures and results for non-uniform lattices}

5.1. A general conjecture. If a group $\Gamma$ acts geometrically on a metric space $X$, then $\Gamma$ is quasi-isometric to $X$. Examples are uniform $\mathrm{S}$-arithmetic groups acting on $X=S \times E \times B$ (see Section 4). This is no longer true for non-uniform S-arithmetic groups like $\mathrm{SL}_{n}\left(\mathbb{Z}\left[\frac{1}{p}\right]\right)$ which in fact can be strongly distorted and thus make the investigation of filling problems considerably more subtle. We posit the following conjectural picture (compare also the discussion in Gromov's book [26], 5D (c)).

Conjecture 1. Let $\Gamma$ be an $S$-arithmetic subgroup of a reductive algebraic group defined over a global field $\mathbb{K}$ such that $\Gamma$ acts as an (irreducible) non-uniform lattice on $X=S \times E \times B$, the associated metric product of symmetric spaces, Euclidean 
spaces and Euclidean buildings. Let $r$ be the Euclidean rank of $X$ and $n$ the dimension of $X$. Then the higher-dimensional Dehn functions of $\Gamma$ satisfy

(i) $\delta_{\Gamma}^{k}(l) \sim l^{\frac{k+1}{k}}$, if $k \leq r-2$.

Moreover, if $\mathbb{K}$ has characteristic zero (i.e., if $\mathbb{K}$ is a number field), then

(ii) $\delta_{\Gamma}^{r-1}(l) \sim \exp l$,

(iii) $\delta_{\Gamma}^{k}(l) \succ l^{\alpha}$ with $1 \leq \alpha$, if $r \leq k \leq n-2$,

(iv) $\delta_{\Gamma}^{n-1}(l) \sim l$.

5.2. Some evidence for Conjecture 1. In the following we discuss partial results which provide some evidence for Conjecture 1.

We will use the elementary

Lemma 4. Let $X$ be a $\mathrm{CAT}(0)$ space and $X_{0}$ a length subspace. Suppose that $C$ is a complete convex subspace both of $X$ and $X_{0}$ in the induced metrics. Then the restriction of the orthogonal projection $\pi: X \rightarrow C$ to $X_{0}$ is 1-Lipschitz.

Proof. By [14], Chapter II, Proposition 2.4 (4), the projection $\pi$ is 1-Lipschitz. Moreover, since $C$ is convex in $X_{0}$ as well as in $X$, we have for all $x, y \in X_{0}$,

$$
\begin{aligned}
\left.d_{X_{0}}\right|_{C}(\pi(x), \pi(y)) & =d_{C}(\pi(x), \pi(y)) \\
& =\left.d_{X}\right|_{C}(\pi(x), \pi(y)) \\
& \leq d_{X}(x, y) \leq d_{X_{0}}(x, y) .
\end{aligned}
$$

5.2.1. Linear Dehn functions in top dimension. The next theorem confirms Conjecture 1(iv) for lattices in semisimple groups.

Theorem 3. Let $X=G / K$ be an $n$-dimensional symmetric space of noncompact type (without Euclidean factor) and let $\Gamma \subset G$ be a lattice. Then the top-dimensional Dehn function of $\Gamma$ is linear:

$$
\delta_{\Gamma}^{n-1}(l) \sim l .
$$

Proof. If $\Gamma$ is uniform the claim follows from Theorem 2. If $\Gamma$ is non-uniform, it does not act cocompactly on $X$, but there is a suitable subspace $X_{0}$ of $X$ obtained by deleting a $\Gamma$-invariant family of horoballs on which $\Gamma$ acts cocompactly (see [23] for $\operatorname{rank} X=1$ and [33] for rank $X \geq 2$ ). Since $\Gamma$ is not virtually solvable (see [39], Chapter 4, Theorem 3.6) it contains a free group by the Tits alternative and thus is nonamenable. Moreover, $X$ and $X_{0}$ have bounded geometry. Thus, by [26], 0.5. $\mathrm{A}_{6}$, the $n$-dimensional (Hausdorff) volume of any domain $\Omega \subset X_{0}$ with $(n-1)$-dimensional measurable boundary $\partial \Omega$ satisfies a linear isoperimetric inequality

$$
\operatorname{vol}_{n}(\Omega) \leq \text { const. } \operatorname{vol}_{n-1}(\partial \Omega)
$$


for a uniform constant depending only on $X_{0}$. This observation yields a linear upper bound for the $n$-dimensional filling function (and hence the $(n-1)$-dimensional Dehn function).

In order to get a linear lower bound for the $n$-dimensional filling function, we choose a geodesic $c$ in $X_{0}$ that is mapped to a closed geodesic in $X / \Gamma$. Such a $c$ exist: One can e.g. choose $c$ in a $\Gamma$-closed flat (see [38], Lemma 8.3'). Then take (a small) $\delta>0$ such that the $\delta$-tube around $c$ is completely contained in $X_{0}$ (replace $X_{0}$ by a $2 \delta$-neighbourhood of $X_{0}$ if necessary). The $\delta$-tube $U_{\delta}\left(c_{l}\right)$ of a segment of $c$ of length $l$ is a convex subset of $X$ and also of $X_{0}$ (see [14], Chapter II, Corollary 2.5). By Lemma 4 the orthogonal projection from $X$ to $U_{\delta}\left(c_{l}\right)$ restricted to $X_{0}$ is 1-Lipschitz. Hence any filling of $\partial U_{\delta}\left(c_{l}\right)$ in $X_{0}$ has at least the same volume as a filling in $U_{\delta}\left(c_{l}\right)$. Since $X$ and $X_{0}$ have bounded geometry, we have

$$
\operatorname{vol}_{n}\left(U_{\delta}\left(c_{l}\right)\right) \sim l \sim \operatorname{vol}_{n-1}\left(\partial U_{\delta}\left(c_{l}\right)\right)
$$

which yields the claimed linear lower bound for the $n$-dimensional filling function.

5.2.2. Proof of Conjecture 1 for real and complex hyperbolic lattices. Here we confirm Conjecture 1 for non-uniform lattices (arithmetic or not) in $\operatorname{SO}(n, 1)$ resp. $\mathrm{SU}(n, 1)$, the group of orientation preserving isometries of real hyperbolic space $H^{n} \mathbb{R}$ resp. complex hyperbolic space $H^{n} \mathbb{C}$. Notice that in this case the Euclidean rank of $X=H^{n} \mathbb{R}$ (resp. $H^{n} \mathbb{C}$ ) is one, so that Conjecture 1 reduces to parts (iii) and (iv). Note further that uniform lattices in $\mathrm{SO}(n, 1)$ resp. $\mathrm{SU}(n, 1)$ are covered by Theorem 2: As the rank is one, all higher-dimensional Dehn functions are linear.

Theorem 4. Let $\Gamma$ be a non-uniform lattice in $\mathrm{SO}(n, 1)$. Then the higher-dimensional Dehn functions of $\Gamma$ satisfy

(a) $\delta_{\Gamma}^{k}(l) \sim l^{\frac{k+1}{k}}$ for $1 \leq k \leq n-2$,

(b) $\delta_{\Gamma}^{n-1}(l) \sim l$.

Proof. It is well known that $\Gamma$ acts geometrically on a CAT(0) space $X_{0}$ obtained from real hyperbolic space $H^{n} \mathbb{R}$ by removing a $\Gamma$-invariant family of disjoint horoballs (see [14], Theorem 11.27, Corollary 11.28). The Euclidean rank of $X_{0}$ is $n-1$ and (isolated) maximal flats are provided by the boundary horospheres of the deleted horoballs. By Wenger's result (Proposition 1) the simply connected CAT $(0)$ space $X_{0}$ satisfies Euclidean isoperimetric inequalities below the rank:

$$
\mathrm{FV}_{X_{0}}^{k+1}(l) \prec l^{\frac{k+1}{k}} \quad \text { for } 1 \leq k \leq n-2,
$$

Lower Euclidean bounds are obtained as follows. Take a maximal flat in $X_{0}$, i.e., a boundary horosphere $H$, and a round $k$-sphere $\Sigma_{k} \subset H$. By Lemma 4 the orthogonal projection from $X_{0}$ to the convex subspace $H$ is 1-Lipschitz. Hence the projection 
of any minimal filling $\Omega_{k+1}$ of $\Sigma_{k}$ in $X_{0}$ orthogonally projects to an even smaller minimal filling $\Omega_{k+1}^{\prime}$ of $\Sigma_{k}$ in $H$ and hence is a $(k+1)$-ball, which yields the Euclidean lower bound:

$$
\operatorname{vol}_{k+1}\left(\Omega_{k+1}\right) \geq \operatorname{vol}_{k+1}\left(\Omega_{k+1}^{\prime}\right) \sim\left(\operatorname{vol}_{k}\left(\Sigma_{k}\right)\right)^{\frac{k+1}{k}} .
$$

Claim (b) on the top-dimensional Dehn function follows from Theorem 3.

Theorem 5. Let $\Gamma$ be a non-uniform lattice in $\mathrm{SU}(n, 1)$. Then the higher-dimensional Dehn functions of $\Gamma$ satisfy

(a) $\delta_{\Gamma}^{k}(l) \sim l^{\frac{k+1}{k}}$ for $1 \leq k \leq n-2$,

(b) $\delta_{\Gamma}^{n-1}(l) \sim l^{\frac{n+1}{n-1}}$,

(c) $\delta_{\Gamma}^{k}(l) \sim l^{\frac{k+2}{k+1}}$ for $n \leq k \leq 2 n-2$,

(d) $\delta_{\Gamma}^{2 n-1}(l) \sim l$.

Proof. As in the proof of Theorem 4 we use the fact that $\Gamma$ acts geometrically on a space $X_{0}$ obtained from real hyperbolic space $H^{n} \mathbb{C}$ by removing a $\Gamma$-invariant family of disjoint horoballs (see e.g. [23] or [33]). Notice that in contrast to the real hyperbolic case, this $X_{0}$ is not a CAT(0) space. The boundary horospheres of $X_{0}$ can be identified with $(2 n-1)$-dimensional Heisenberg groups $H_{2 n-1}$. Moreover, given a boundary horosphere $\mathscr{H} \subset \partial X_{0}$, there is a 1-Lipschitz retraction $r: X_{0} \rightarrow \mathscr{H}$ (see [33]). Hence, if $\Sigma$ is a $k$-cycle in $\mathscr{H}$ any filling $\Omega$ of $\Sigma$ in $X_{0}$ cannot be of smaller volume than that of the minimal filling of $\Sigma$ in $\mathscr{H}$. This yields lower bounds for the filling functions of $X_{0}$ :

$$
\mathrm{FV}_{X_{0}}^{k+1} \succ \mathrm{FV}_{\mathcal{H}}^{k+1} \text { for } 1 \leq k \leq 2 n-2 .
$$

In order to obtain upper bounds we argue as in [26], 5.D.5 (c). Given a $k$-cycle $\Sigma$ in $X_{0}$ we first take a filling $\widetilde{\Omega}$ of $\Sigma$ in $X$ with $\operatorname{vol}_{k+1}(\widetilde{\Omega}) \prec \operatorname{vol}_{k}(\Sigma)$ (such a linear filling exists by Theorem 1). Then we consider the intersection $\tilde{\Sigma}:=\widetilde{\Omega} \cap \partial X_{0}$. This is a $k$-cycle in $\partial X_{0}$. We fill $\widetilde{\Sigma}$ by a minimal $(k+1)$-chain $\hat{\Omega}$ in $\partial X_{0}$. Together with $\widetilde{\Omega} \cap X_{0}$ this eventually yields a $(k+1)$-chain $\Omega$ which fills $\Sigma$ in $X_{0}$.

Now assume that $\operatorname{vol}_{k}(\Sigma)=l$. By taking a $\epsilon$-neighbourhood of $\widetilde{\Sigma}$ we see that

$$
\operatorname{vol}_{k}(\tilde{\Sigma}) \sim \operatorname{vol}_{k+1}\left(U_{\epsilon}(\tilde{\Sigma}) \cap \tilde{\Omega}\right)<\operatorname{vol}_{k+1}(\tilde{\Omega}) \prec \operatorname{vol}_{k}(\Sigma) .
$$

Hence also have $\operatorname{vol}_{k}(\tilde{\Sigma}) \prec l$. This yields

$$
\begin{aligned}
\operatorname{vol}_{k+1}(\Omega) & \leq \operatorname{vol}_{k+1}\left(\tilde{\Omega} \cap X_{0}\right)+\operatorname{vol}_{k+1}(\widehat{\Omega}) \\
& \leq \operatorname{vol}_{k+1}(\tilde{\Omega})+\operatorname{vol}_{k+1}(\hat{\Omega}) \leq l+\mathrm{FV}_{\partial X_{0}}^{k+1}(l),
\end{aligned}
$$


where the last estimate follows from the fact that $\widehat{\Omega}$ is a minimal filling in $\partial X_{0}$ of the cycle $\widetilde{\Sigma}$, which by the above also has $k$-volume $\leq l$. Since $\partial X_{0}$ is the union of disjoint horospheres we further have $\mathrm{FV}_{\partial X_{0}}^{k+1} \sim \mathrm{FV}_{\mathscr{H}}^{k+1}$. We conclude that

$$
\mathrm{FV}_{X_{0}}^{k+1}(l) \leq l+\mathrm{FV}_{\mathscr{H}}^{k+1}(l) \sim l+\mathrm{FV}_{H_{2 n-1}}^{k+1}(l) .
$$

The higher-dimensional filling functions of Heisenberg groups have been determined by Young in [51]. As they are superlinear we obtain that

$$
\mathrm{FV}_{X_{0}}^{k+1} \prec \mathrm{FV}_{H_{2 n-1}}^{k+1} \sim \mathrm{FV}_{\mathscr{H}}^{k+1}
$$

Together with the lower bound above this eventually yields for the filling functions of $X_{0}$ :

$$
\mathrm{FV}_{X_{0}}^{k+1} \sim \mathrm{FV}_{\mathcal{H}}^{k+1} \sim \mathrm{FV}_{H_{2 n-1}}^{k+1} \text { for } 1 \leq k \leq 2 n-2 .
$$

As $\Gamma$ is quasi-isometric to $X_{0}$ we get for the Dehn functions for $\Gamma$ :

$$
\delta_{\Gamma}^{k} \sim \delta_{X_{0}}^{k} \sim \delta_{\mathscr{H}}^{k} \sim \delta_{H_{2 n-1}}^{k} \text { for } 1 \leq k \leq 2 n-2 .
$$

Parts (a), (b) and (c) of the theorem then follow from [51].

Claim (d) on the linear top-dimensional Dehn function follows from Theorem 3.

5.2.3. Low dimensional Euclidean lower bounds. The last argument of the proof of Theorem 4 (a) generalizes to non-uniform lattices of semisimple Lie groups:

Theorem 6. Let $\Gamma$ be an irreducible, non-uniform lattice in a semisimple Lie group $G$ acting on a symmetric space $X=G / K$ of rank $r$. Then the Dehn functions of dimension between 1 and $r-2$ have Euclidean lower bounds:

$$
\delta_{\Gamma}^{k}(l) \succ l^{\frac{k+1}{k}} \text { if } 1 \leq k \leq r-2 .
$$

Proof. A theorem of Mostow asserts that there are many closed maximal flats in $X \backslash \Gamma$, see [38], Lemma 8.3, 8.3'. Pick one such flat and its universal cover $F$ in $X_{0}$ (a length subspace of $X$ quasi-isometric to $\Gamma$, see [23] and [33]). Then the restriction of the orthogonal projection $\pi: X \rightarrow F$ to $X_{0}$ is 1-Lipschitz by Lemma 4 and the claim follows from the solution of the Euclidean isoperimetric problem in $F$.

5.2.4. Exponential (r-1)-dimensional Dehn functions. Conjecture 1 (ii), i.e., that the $(r-1)$-dimensional Dehn function is exponential, has been proved in a number of cases.

By a result of Gromov (see [33] for a detailed proof) Dehn functions (of any dimension) of linear groups are at most exponential.

Wortman, in [48], established lower exponential bounds for many non-uniform arithmetic subgroups of semisimple algebraic $\mathbb{Q}$-group. The cases where the conjecture for such groups is still open are those of relative $\mathbb{Q}$-type $B C_{n}, G_{2}, F_{4}$ and $E_{8}$. 
In [34] we proved exponential lower bounds for the 1-dimensional Dehn function of (irreducible) non-uniform lattices in all semisimple Lie groups of rank 2.

By Taback [45], the 1-dimensional Dehn function for the (simplest) $S$-arithmetic group $\operatorname{PSL}_{2}\left(\mathbb{Z}\left[\frac{1}{p}\right]\right), p$ a prime number, is exponential.

5.3. The Bux-Wortman conjecture. A unified approach to large scale properties of $S$-arithmetic groups on function fields or number fields has been formulated in a conjecture by Bux and Wortman [17]. It is based on a geometric version of reduction theory and uses the concept of coarse manifolds to measure the distorsion of the metrics in various dimensions.

A coarse $n$-manifold of scale $s$ in a metric space $X$ is the image of a map $C: M \rightarrow$ $X$ from a triangulated $n$-dimensional manifold $M$ (with boundary) such that any two adjacent vertices of the triangulation are mapped to points of distance at most $s$ in $X$. The boundary of a coarse manifold is the image under $C$ of the boundary of $M$. The volume of a coarse manifold is defined as the number of vertices in the triangulation. A subset $Y \subset X$ is called (homotopically) undistorted in dimension $k$, if there are positive constants $s_{1}$ and $s_{2}$ and a linear function $L: \mathbb{R} \rightarrow \mathbb{R}$ such that the following holds:

For any coarse $k$-ball $B$ in $X$ of scale at most $s_{1}$ whose boundary is a coarse sphere in $Y$, there is a coarse $k$-ball $B^{\prime}$ in $Y$ of scale at most $s_{2}$ with the same boundary, such that $\operatorname{vol}_{k}\left(B^{\prime}\right) \leq L\left(\operatorname{vol}_{k}(B)\right)$.

The (homotopical) distorsion dimension of $Y$ in $X$ is the largest dimension $k$ such that $Y$ is homotopically undistorted in dimension $k$.

Consider now a reductive $\mathbb{K}$-group $\boldsymbol{G}$ defined over a global field $\mathbb{K}$ and a finite set $S$ of valuations as in Section 4.1. Let $\boldsymbol{G}_{S}$ be the semisimple Lie group $\boldsymbol{G}_{S}=\prod_{v \in S} \boldsymbol{G}\left(\mathbb{K}_{v}\right)$ endowed with a left-invariant metric. Notice that this metric space is quasi-isometric to the product $X=S \times B$ of a symmetric space and Euclidean buildings on which $\boldsymbol{G}_{S}$ acts isometrically. The metric on $\boldsymbol{G}_{S}$ restricts to the diagonally embedded lattice $\boldsymbol{G}\left(\mathcal{O}_{S}\right)$. The Euclidean rank $\boldsymbol{r}$ of $\boldsymbol{G}_{S}$ (or $\left.X\right)$ is the sum of the local ranks $r:=\sum_{s \in S} \operatorname{rank}_{\mathbb{K}_{v}} \boldsymbol{G}$.

Conjecture 2 (Bux-Wortman, [17]). The $S$-arithmetic subgroup $\boldsymbol{G}\left(\mathcal{O}_{S}\right)$ has distortion dimension $r-1$ as a subgroup of the reductive group $\boldsymbol{G}_{S}$ with respect to the metrics defined above.

As mentioned above, $S$-arithmetic groups over number fields are of type $F_{\infty}$. In contrast, $S$-arithmetic groups over function fields are almost never of type $F_{\infty}$.

Conjecture 1 (ii) implies that the upper bound in Conjecture 2 is sharp also in the number field case. This indicates that are deep similarities from the point of view of coarse geometry between $S$-arithmetic groups over function fields and those over number fields: In both cases the distortion dimension should be equal to the Euclidean rank minus 1 . 
A weakened and special case of Conjecture 2 has recently been established in [6]. Further evidence comes from the solution of Thurston's conjecture, that $\mathrm{SL}_{n}(\mathbb{Z})$ has a quadratic 1-dimensional Dehn function, by Young (see [50] for $n \geq 5$ ). Work of Lubotzky-Mozes-Raghunathan [35] asserts that, for $r \geq 2, \boldsymbol{G}\left(\mathcal{O}_{S}\right)$ is undistorted in $\boldsymbol{G}_{S}$ in dimension 1. Since this is not the case for $r=1$, it follows that $\operatorname{dist-\operatorname {dim}}\left(\boldsymbol{G}\left(\mathcal{O}_{S}\right)\right)=0$ if and only if the Euclidean rank of $\boldsymbol{G}_{S}$ is 1 . Moreover, results of Leuzinger-Pittet [34] (for number fields), Behr [5] (for function fields) and Taback [45] (for $\left.\operatorname{PSL}_{2}\left(\mathbb{Z}\left[\frac{1}{p}\right]\right)\right)$ show that dist-dim $\left(\boldsymbol{G}\left(\mathcal{O}_{S}\right)\right)=1$, if the Euclidean rank of $\boldsymbol{G}_{S}$ is 2 .

Finally, we remark that in view of Theorem 1 and Theorem 6, in order to prove Conjecture 2, the problem is to establish Euclidean isoperimetric inequalities below the rank.

Acknowledgement. I thank Robert Young for pointing out to me some inaccuracies in an earlier version of this paper.

\section{References}

[1] A. Abrams, N. Brady, P. Dani, M. Duchin, and R. Young, Pushing fillings in rightangled Artin groups. J. London Math. Soc. (2) 87 (2013), no. 3, 663-688. Zbl 06188918 MR 3073670

[2] J. M. Alonso, Finiteness conditions on groups and quasi-isometries. J. Pure Appl. Algebra 95 (1994), 121-129. Zbl 0823.20034 MR 1293049

[3] J. M. Alonso, X. Wang, and S. J. Pride, Higher-dimensional isoperimetric (or Dehn) functions of groups. J. Group Theory 2 (1999), 81-122. Zbl 0927.20021 MR 1670329

[4] A. Avez, Variétés Riemanniennes sans points focaux. C. R. Acad. Sci. Paris 270 (1970), 188-191. Zbl 0188.26402 MR 0256305

[5] H. Behr, Arithmetic groups over function fields I. J. Reine Angew. Math. 495 (1998), 79-118. Zbl 0923.20038 MR 1603845

[6] M. Bestvina, A. Eskin, and K. Wortman, Filling boundaries of coarse manifolds in semisimple and solvable arithmetic groups. J. Eur. Math. Soc. (JEMS) 15 (2013), no. 6, 2165-2195. Zbl 06243071 MR 3120741

[7] A. Borel, Compact Clifford-Klein forms of symmetric spaces. Topology 2 (1963), 111-122. Zbl 0116.38603 MR 0146301

[8] A. Borel and Harish-Chandra, Arithmetic subgroups of algebraic groups. Ann. of Math. (2) 75 (1962), 485-535. Zbl 0107.14804 MR 0147566

[9] A. Borel and J.-P. Serre, Cohomologie d'immeubles et de groupes $S$-arithmétiques. Topology 15 (1967), 211-232. Zbl 0338.20055 MR 0447474

[10] A. Borel and G. Harder, Existence of discrete cocompact subgroups of reductive groups over local fields. J. Reine Angew. Math. 298 (1978), 53-64. Zbl 0385.14014 MR 0483367

[11] N. Brady, M. R. Bridson, M. Forester, and K. Shankar, Snowflake groups, PerronFrobenius eigenvalues and isoperimetric spectra. Geom. Topol. 13 (2009), 141-187. Zbl 1228.20031 MR 2469516 
[12] M. R. Bridson, The geometry of the word problem. In Invitations to geometry and topology, M. R. Bridson and S. M. Salamon, eds., Oxf. Grad. Texts Math. 7, Oxford University Press, Oxford 2002, 29-91. Zbl 0996.54507 MR 1967746

[13] M. R. Bridson, Polynomial Dehn functions and the length of asynchronously automatic structures. Proc. London Math. Soc. (3) 85 (2002), 441-466. Zbl 1046.20027 MR 1912057

[14] M. R. Bridson and A. Haefliger, Metric spaces of non-positive curvature. Grundlehren Math. Wiss. 319, Springer, Berlin 1999. Zbl 0988.53001 MR 1744486

[15] D. Burago, I. Burago, and S. Ivanov, A course in metric geometry. Grad. Stud. Math. 33, Amer. Math. Sooc., Providence, RI, 2001. Zbl 0981.51016 MR 1835418

[16] D. Burago and V. Zalgaller, Geometric inequalities. Grundlehren Math. Wiss. 285, Springer Ser. in Soviet Math., Springer, Berlin 1988. Zbl 0633.53002 MR 0936419

[17] K.-U. Bux and K. Wortman, Finiteness properties of arithmetic groups over function fields. Invent. Math. 167 (2007), 355-378. Zbl 1126.20030 MR 2270455

[18] K.-U. Bux, R. Gramlich, and S. Witzel, Higher finiteness properties of reductive arithmetic groups in positive characteristic: the rank theorem. Ann. of Math. (2) 177 (2013), no. 1, 311-366. Zbl 06146421 MR 2999042

[19] P. Eberlein, Geometry of nonpositively curved manifolds. Chicago Lectures in Math., University of Chicago Press, Chicago, IL, 1996. Zbl 0883.53003 MR 1441541

[20] D. B. A. Epstein et al., Word processing in groups. Jones and Barlett Publishers, Boston, MA, 1992. Zbl 0764.20017 MR 1161694

[21] H. Federer, Geometric measure theory. Grundlehren Math. Wiss. 153, Springer, New York 1969. Zbl 0176.00801 MR 0257325

[22] F. R. Gantmacher, Theory of matrices. Vol. 1. AMS Chelsea Publishing, Providence, RI, 1998. Zbl 0927.15001 MR 1657129

[23] H. Garland and M. S. Raghunathan, Fundamental domains for lattices in $R$-rank 1 Lie groups. Ann. of Math. (2) 92 (1970), 279-326. Zbl 0206.03603 MR 0267041

[24] C. Groft, Generalized Dehn functions I, II. Preprint, arXiv:0901.2303, arXiv:0901.2317.

[25] M. Gromov, Filling Riemannian manifolds. J. Differential Geom. 18 (1983), 1-147. Zbl 0515.53037 MR 0697984

[26] M. Gromov, Asymptotic invariants of infinite groups. In Geometric group theory, Vol. 2, G. A. Niblo and M. A. Roller, eds., London Math. Soc. Lecture Note Ser. 182, Cambridge University Press, Cambridge 1993, 1-295. Zbl 0841.20039 MR 1253544

[27] M. Gromov, Metric structures for Riemannian and non-Riemannian spaces. Progr. Math. 152, Birkhäuser, 1999. Zbl 0953.53002 MR 1699320

[28] V. Guillemin and A. Pollack, Differential topology. Prentice Hall, Englewood Cliffs, NJ, 1974. Zbl 0361.57001 MR 0348781

[29] G. Harder, Über die Galoiskohomologie halbeinfacher algebraischer Gruppen, III. $J$. Reine Angew. Math. 274/275 (1975), 125-138. Zbl 0317.14025 MR 0382469

[30] S. Helgason, Differential geometry, Lie groups, and symmetric spaces. Pure Appl. Math. 80, Academic Press, London 1978. Zbl 0451.53038 MR 0514561 
[31] P. Kropholler and G. Mislin, Groups acting on finite-dimensional spaces with finite stabilizers. Comment. Math. Helv. 73 (1998), 122-136. Zbl 0927.20033 MR 1610595

[32] U. Lang, Higher-dimensional linear isoperimetric inequalities in hyperbolic groups. Internat. Math. Res. Notices 13 (2000), 709-717. Zbl 0982.20027 MR 1772520

[33] E. Leuzinger, On polyhedral retracts and compactifications of locally symmetric spaces. Differential Geom. Appl. 20 (2004), 293-318. Zbl 1052.22008 MR 2053916

[34] E. Leuzinger and Ch. Pittet, Isoperimetric inequalities for lattices in semisimple Lie groups of rank 2. Geom. Funct. Anal. 6 (1996), 489-511. Zbl :0856.22013 MR 1392327

[35] A. Lubotzky, S. Mozes, and M. S. Raghunathan, The word and Riemannian metrics on lattices of semisimple groups. Inst. Hautes Études Sci. Publ. Math. 91 (2000), 5-53. Zbl 0988.22007 MR 1828742

[36] G. A. Margulis, Discrete subgroups of semisimple Lie groups. Ergeb. Math. Grenzgeb. (3) 17, Springer, Berlin 1991. Zbl 0732.22008 MR 1090825

[37] I. Mineyev, Higher dimensional isoperimetric functions in hyperbolic groups. Math. Z. 233 (2000), 327-345. Zbl 0954.20021 MR 1743440

[38] G. D. Mostow, Strong rigidity of locally symmetric spaces. Ann. of Math. Stud. 78, Princeton University Press, Princeton, NJ, 1973. Zbl 0265.53039 MR 0385004

[39] A. L. Onishchik and E. B. Vinberg (eds.), Lie Groups and Lie algebras II. Encyclopaedia Math. Sci. 21, Springer, Berlin 2000. Zbl 0932.00011 MR 1756406

[40] M. S. Raghunathan, A note on quotients of real algebraic groups by arithmetic subgroups. Invent. Math. 4 (1967), 318-335. Zbl 0218.22015 MR 0230332

[41] T. Riley, Higher connectedness of asymptotic cones. Topolgy 42 (2003), 1289-1352. Zbl 1038.20031 MR 1981358

[42] T. Sakai, Riemannian geometry. Transl. Math. Monogr. 149, Amer. Math. Soc., Providence, RI, 1996. Zbl 0886.53002 MR 1390760

[43] J.-P. Serre, Cohomologie des groupes discrets. In Prospects in Mathematics, Ann. of Math. Stud. 70, Princeton University Press, Princeton, NJ., 1971, 77-169. Zbl 0235.22020 MR 0385006

[44] T. A. Springer, Reductive groups. In Automorphic forms, representations and Lfunctions, Proc. Symp. Pure Math. 33, Amer. Math. Soc., Providence, RI, 1979, 3-27. Zbl 0416.20034 MR 0546587

[45] J. Taback, The Dehn function of $\operatorname{PSL}_{2}\left(\mathbb{Z}\left[\frac{1}{p}\right]\right)$. Geom. Dedicata 102 (2003), 179-195. Zbl 1039.20021 MR 2026844

[46] E. B. Vinberg (ed.), Geometry II, Encyclopaedia Math. Sci. 29, Springer, Berlin 1993. Zbl 0786.00008 MR 1254931

[47] S. Wenger, Isoperimetric inequalities of Euclidean type in metric spaces. Geom. Funct. Anal. 15 (2005), 534-554. Zbl 1084.53037 MR 2153909

[48] K. Wortman, Exponential higher dimensional isoperimetric inequalities for some arithmetic groups. Geom. Dedicata 151 (2011), 141-153. Zbl 1219.20027 MR 2780742

[49] R. Young, Homological and homotopical higher-order filling functions. Groups Geom. Dyn. 5 (2011), 683-690. Zbl 1263.20040 MR 2813532 
[50] R. Young, The Dehn function of $\mathrm{SL}_{n}(\mathbb{Z})$. Ann. of Math. (2) 177 (2013), no. 3, 969-1027. Zbl 1276.20053 MR 3034292

[51] R. Young, High-dimensional fillings in Heisenberg groups. Preprint, arXiv:1006.1636v1.

[52] R. Young, Filling inequalities for nilpotent groups through approximations. Groups Geom. Dyn. 7 (2013), no. 4, 977-1011. Zbl 06256450 MR 3134033

Received August 7, 2012; revised January 21, 2013

E. Leuzinger, Institute for Algebra und Geometry, Karlsruhe Institute of Technology (KIT), 76133 Karlsruhe, Germany

E-mail: enrico.leuzinger@kit.edu 